\title{
Who Are Controlled by The Same User? Multiple Identities Deception Detection via Social Interaction Activity (Student Abstract)
}

\author{
Jiacheng Li, ${ }^{1,2}$ Chunyuan Yuan, ${ }^{1,2}$ Wei Zhou, ${ }^{2, *}$ Jingli Wang, ${ }^{3}$ Songlin $\mathbf{H u}^{2}$ \\ ${ }^{1}$ School of Cyber Security, University of Chinese Academy of Sciences, Beijing, China \\ ${ }^{2}$ Institute of Information Engineering, Chinese Academy of Sciences, Beijing, China \\ ${ }^{3}$ China Government Securities Depository Trust \& Clearing Co., Ltd., Shanghai, China \\ \{lijiacheng, yuanchunyuan, zhouwei, wangjingli, husonglin\}@iie.ac.cn
}

\begin{abstract}
Social media has become a preferential place for sharing information. However, some users may create multiple accounts and manipulate them to deceive legitimate users. Most previous studies utilize verbal or behavior features based methods to solve this problem, but they are only designed for some particular platforms, leading to low universalness.

In this paper, to support multiple platforms, we construct interaction tree for each account based on their social interactions which is common characteristic of social platforms. Then we propose a new method to calculate the social interaction entropy of each account and detect the accounts which are controlled by the same user. Experimental results on two real-world datasets show that the method has robust superiority over state-of-the-art methods.
\end{abstract}

\section{Introduction}

Multiple identities (sockpuppets) detection is a critical issue in social media. Previous studies (Kumar et al., 2017; Wang et al., 2018) have shown that sockpuppets are often applied to manipulate public opinion and heavily affect the user experience.

Recent studies focus on profile similarity based (Keselj et al., 2003; Li et al., 2017), verbal based (Solorio, Hasan, and Mizan, 2013) and behavior features based (Tsikerdekis and Zeadally, 2014) methods to detect the sockpuppets. Profile similarity based methods use the account profile, such as nickname (Keselj et al., 2003) and description of account (Li et al., 2017), for detection. Verbal based methods assume that sockpuppets have similar linguistic traits $(\mathrm{Ku}-$ mar et al., 2017) and extract text and semantic features from posts and comments. However, smart malicious users could change nicknames and writing style to disguise themselves.

Thus, behavior feature based methods are proposed to analyze account behaviors, such as the total number of bytes removed from all the revisions (Tsikerdekis et al,2014), cooccurrence relationship (Liu et al., 2016). However, previous methods have high dependence on the particular platforms, thus lack generalization. For example, as a video plat-

\footnotetext{
${ }^{*}$ Wei Zhou is corresponding author Copyright (c) 2020, Association for the Advancement of Artificial Intelligence (www.aaai.org). All rights reserved.
}

form, TikTok can hardly provide useful text to extract verbal features. In this paper, we propose a platform-independent interaction entropy method based on the social interaction activity. The method can be easily applied to many social media platforms.

The contribution of this work can be concluded as follows: (1) We apply the interaction activities, which are available on mainstream social platforms, to extract general features that are easily available from many platforms. (2) We propose a novel interaction entropy algorithm to depict the interaction activities of users for sockpuppets detection. (3) The experimental results achieve a significant performance over state-of-the-art methods and validate the effectiveness of our method.

\section{Methodology}

We extract the interaction activity from interaction logs and construct interaction tree as shown in Figure 1. Then we propose the interaction entropy based on the interaction tree inspired by the ideal of the graph entropy (Dehmer, 2008). Firstly, we define user interaction set $D_{u}=\left\{d_{i}^{u} \mid u \in U, i \in\right.$ $M$ \}, where $d_{i}^{u}$ is the interaction tree of account $u$ based on message $i, U$ is the set of users and $M$ denotes the messages in the social network. The function $\left|S\left(v, d_{i}^{u}\right)\right|$ denotes the depth of node $v$ in $d_{i}^{u}$ as shown in Figure 2. For a node $v \in$ $d_{i}^{u}$, we define the node entropy function:

$$
f(v)=\left|S\left(v, d_{i}^{u}\right)\right| \cdot \frac{C(v)}{C\left(d_{i}^{u}\right)},
$$

where $C(v)$ is the number of account $v$ in $d_{i}^{u}$ (an account may appear many times in one tree) and $C\left(d_{i}^{u}\right)$ is the number of nodes in the tree $d_{i}^{u}$. Then we propose an function to represent the entropy of the interaction tree $d_{i}^{u}$.

$$
H_{p}\left(d_{i}^{u}\right)=\sum_{i=0}^{C\left(d_{i}^{u}\right)}-p\left(v, d_{i}^{u}\right) \cdot \log p\left(v, d_{i}^{u}\right),
$$

where $p\left(v, d_{i}^{u}\right)=\frac{f(v)}{\sum_{i=0}^{C\left(d_{i}^{u}\right)} \alpha^{f(v)}}$ is the probability mass function for the tree. The hyperparameters $\alpha$ is a shrinkage factor. According to the above definition, a tree has more diverse interactions when it has a lower entropy value. 
Table 1: Multiple Identity Deception Detection

\begin{tabular}{|c|c|c|c|c|c|c|}
\hline \multirow{2}{*}{ Method } & \multicolumn{3}{|c|}{$\mathcal{D}_{\mathcal{S}}$} & \multicolumn{3}{c|}{$\mathcal{D}_{\mathcal{T}}$} \\
\cline { 2 - 7 } & Precision & Recall & F1-Score & Precision & Recall & F1-Score \\
\hline Verbal Based (Solorio et al., 2013) & 0.755 & 0.580 & 0.645 & 0.701 & 0.696 & 0.697 \\
Behavior Features Based (Tsikerdekis et al., 2014) & 0.700 & 0.564 & 0.622 & 0.766 & 0.765 & 0.764 \\
Behavior Features Based (Liu et al., 2016) & 0.680 & 0.700 & 0.690 & 0.760 & 0.580 & 0.660 \\
Profile Similarity Based (Li et al., 2017) & 0.746 & 0.686 & 0.707 & 0.710 & 0.672 & 0.690 \\
Behavior Features Based (Wang et al., 2018) & 0.840 & 0.870 & 0.850 & 0.830 & 0.800 & 0.820 \\
Our Method & $\mathbf{0 . 8 6 0}$ & $\mathbf{0 . 8 9 0}$ & $\mathbf{0 . 8 7 5}$ & $\mathbf{0 . 9 0 0}$ & $\mathbf{0 . 8 9 4}$ & $\mathbf{0 . 8 9 7}$ \\
\hline
\end{tabular}

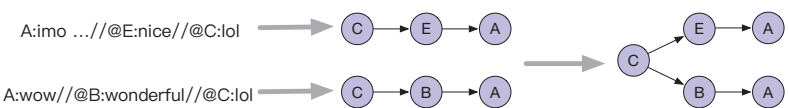

Figure 1: Interaction Tree Construction Process. We extract the interaction link list from interaction logs and merge the link list with the same head such as username and tweet.

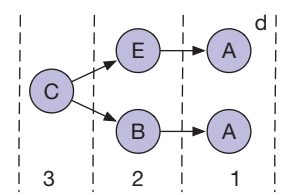

Figure 2: The depth of interaction tree, for example $\left|S\left(B, d_{i}^{u}\right)\right|=2$. The depth is an integer starting with 1 at the deepest leaf node for the convenience of calculation.

Then, we obtain an interaction entropy of account $u$ which indicates interaction diversity.

$$
I_{f}(u)=\sum_{i=0}^{\left|D_{u}\right|}-H_{p}\left(d_{i}^{u}\right) \cdot \log _{2} H_{p}\left(d_{i}^{u}\right),
$$

where $\left|D_{u}\right|$ is the size of set $D_{u}$.

Based on the interaction entropy, we show the multiple identities deception detection algorithm. Given accounts $u$ and $v$, we will get the function $\mathrm{G}$ to depict the social interaction activity difference, which can be formalized as follows:

$$
G(u, v)=\left|\frac{I_{f}(u)}{I_{f}(v)}-1.0\right| .
$$

Finally, we can obtain the result to discriminate for multiple identities via the function $\mathrm{G}$.

$$
G(u, v)= \begin{cases}\leqslant \beta & \mathrm{u}, \mathrm{v} \text { are controlled by the same user } \\ >\beta & \mathrm{u}, \mathrm{v} \text { belong to different users }\end{cases}
$$

where $\beta$ is a threshold and the accounts whose difference score below the $\beta$ is controlled by the same malicious user.

\section{Experiments and Result}

Datasets. In this paper, we conduct experiments on two realworld datasets $\mathcal{D}_{\mathcal{S}}$ and $\mathcal{D}_{\mathcal{T}}$ which are public available at (Wang et al., 2018). The $\mathcal{D}_{\mathcal{S}}$ dataset contains 675 users. The
$\mathcal{D}_{\mathcal{T}}$ dataset contains 991 users. Accounts are identified as sockpuppets according to rules, such as self-reported sentence matching like 'This is a sockpuppet of Mix'.

Experiments In the experiments, we set the model parameters as: $\alpha=2.3, \beta=0.6$. The analyses of parameter sensitivity can be found in the supplemental materials. As shown in the Table 1, our model achieves significantly improvement over state-of-the-art methods on two datasets $(+2.5$ $30.5 \%$ in terms of F1), which shows the effectiveness of the model. Furthermore, the interaction relations are easily available on mainstream social media platforms, such as Twitter, Facebook, Weibo, etc., thus the algorithm has wider applications compared with previous methods.

\section{Conclusion}

In this paper, we study the multiple identities deception detection problem, with the platform independent model based on social interaction structure. Then we raise a novel interaction entropy algorithm to depict the interaction activity. The experimental results validate the effectiveness of our method on two real-world datasets.

\section{References}

Dehmer, M. 2008. Information processing in complex networks: Graph entropy and information functionals. Applied Mathematics and Computation 201(1-2):82-94.

Keselj, V.; Peng, F.; Cercone, N.; and Thomas, C. 2003. N-grambased author profiles for authorship attribution. In PACLING'O3, 255-264.

Kumar, S.; Cheng, J.; Leskovec, J.; and Subrahmanian, V. S. 2017. An army of me: Sockpuppets in online discussion communities. In $W W W^{\prime} 17,857-866$.

Li, Y.; Peng, Y.; Ji, W.; Zhang, Z.; and Xu, Q. 2017. User identification based on display names across online social networks. IEEE Access 5:17342-17353.

Liu, D.; Wu, Q.; Han, W.; and Zhou, B. 2016. Sockpuppet gang detection on social media sites. Frontiers Comput. Sci. 10(1):124135.

Solorio, T.; Hasan, R.; and Mizan, M. 2013. A case study of sockpuppet detection in wikipedia. In Proceedings of the Workshop on Language Analysis in Social Media, 59-68.

Tsikerdekis, M., and Zeadally, S. 2014. Multiple account identity deception detection in social media using nonverbal behavior. IEEE Trans. Information Forensics and Security 9(8):1311-1321.

Wang, J.; Zhou, W.; Li, J.; Yan, Z.; Han, J.; and Hu, S. 2018. An online sockpuppet detection method based on subgraph similarity matching. In ISPA'18, 391-398. 